\title{
Efecto del Salvado de Trigo en el Comportamiento Reológico del Yogurt de Leche de Búfala
}

\author{
Ricardo D. Andrade*, Margarita R. Arteaga y Mónica M. Simanca \\ Universidad de Córdoba, Programa de Ingeniería de alimentos, Grupo Investigaciones en procesos \\ agroindustriales, $\mathrm{Km} 12$ Vía Cereté, Ciénaga de Oro-Colombia \\ (e-mail: randrade@sinu.unicordoba.edu.co, ricardoandradep@yahoo.com) \\ *autor a quien debe ser dirigida la correspondencia
}

Recibido May. 07, 2009; Aceptado Jul. 10, 2009; Versión Final recibida Sep. 03, 2009

\section{Resumen}

En el presente estudio se evaluó el efecto de la adición de salvado de trigo $(0,1,3,5 \% \mathrm{p} / \mathrm{v})$ y el tiempo de almacenamiento en el comportamiento reológico del yogurt de leche de búfala. A la leche de búfala se le realizaron análisis de materia grasa, presencia de antibiótico, sólidos totales, acidez y $\mathrm{pH}$. Para las medidas reológicas se utilizó un viscosímetro Brookfied modelo DV-II+Pro, ajustando los datos al modelo de Oswald de Wale. El yogurt presentó un comportamiento seudoplástico y tixotrópico. La adición de salvado de trigo influyó de manera directamente proporcional sobre los parámetros reológicos, haciéndolo más consistente y menos seudoplástico. El tiempo de almacenamiento lo hizo de manera inversamente proporcional al índice de consistencia y directamente proporcional al índice de flujo, siendo menos consistente y con una tendencia a fluido newtoniano.

Palabras clave: yogurt, leche de búfala, reología, Ostwald de Waele, seudoplástico, tixotrópico

\section{Effect of the bran wheat on the rheological behavior of yogurt buffalo milk}

\begin{abstract}
In the present study the effect of wheat bran addition $(0,1,3,5 \% \mathrm{w} / \mathrm{v})$ and the storage time in the rheological behavior of the yogurt buffalo milk were evaluated. Fat matter, antibiotic presence, total solids, acidity and $\mathrm{pH}$ were determined in buffalo milk. Rheological measurements were carried out using a Brookfied viscometer DV-II+Pro model, fitting the data to the model of Oswald de Wale. The yogurt presented a pseudoplastic and thixotropic behavior. Wheat bran addition influenced proportional directly way the rheological parameters, making more consistent and less pseudoplastic. Storage time was inversely proportional to the consistency index and directly proportional to the flow index, being less consistent, with tendency to Newtonian fluid.
\end{abstract}

Keywords: yogurt, buffalo milk, rheology, Ostwald de Waele, pseudoplastic, thixotropic 


\section{INTRODUCCIÓN}

La leche de búfala presenta características muy propias que permiten su fácil identificación desde el punto de vista físico-químico y organoléptico; su sabor es peculiar, levemente endulzado y de color blanco, debido a la ausencia casi total de carotenos en su grasa; presenta niveles mayores de grasa, sólidos totales, calorías, vitamina A y calcio en relación con la leche de vaca (Guarín, 2002; Verruma y Salgado, 1994). La leche de búfala por su composición puede ser utilizada como materia prima para la elaboración de productos lácteos, tales como quesos, leche en polvo, leches maternizadas, leches fermentadas, helados, dulce de leche, entre otros y además posee un óptimo rendimiento en la elaboración de los mismos, ya que tiene mas sólidos totales, grasa, proteína y lactosa que la leche bovina (Silva et al., 2003; Patiño, 2004).

El yogurt es un alimento obtenido de la fermentación controlada de la leche, por las acciones combinadas de cultivos de bacterias ácido lácticas, las cuales provocan un sabor láctico y aroma típico. En la actualidad, el yogurt y sus ingredientes, así como sus propiedades, han sido motivo de muchos estudios, tanto bromatológicos, clínicos, microbiológicos, nutricionales, como ingenieriles (Vélez y Rivas, 2001; Tamine y Robinson, 1991).

La incorporación de nuevos ingredientes en la formulación del yogurt, cambia la estructura original del gel tanto física como químicamente, por lo que es importante conocer sus efectos. Se ha tratado de enriquecer al yogurt mediante la adición de fibra, vitaminas, y calcio, entre otros nutrientes, convirtiéndolo en un alimento funcional (Díaz et al., 2004; Saxelin et al., 2003). El aumento del contenido de fibra, en el yogurt, tiene como consecuencia un aumento del coeficiente de consistencia e incremento de la pseudoplasticidad; el contenido de grasa también afecta estos parámetros reológicos y el tiempo de almacenamiento afecta todos los factores arriba mencionados, lo que básicamente refleja la pérdida de calidad que sufre el yogurt durante su vida de anaquel (Díaz et al., 2004). El yogurt con una adición hasta con un $30 \%$ de suero de leche presenta un comportamiento dependiente del tiempo y una disminución del índice de consistencia (Gauche et al., 2009).

Sistemas modelos con hidrocoloides se ajustan al modelo de ley potencia, presentando un área entre las curvas de ascenso y descenso de los reogramas, está histéresis indica el comportamiento tixotrópico de las muestras (Razavi y Karazhiyan, 2009). El enriquecimiento de helados con fibra es un manera eficaz de mejorar los aspectos nutricionales y de influir en la funcionalidad de las propiedades reológicas y térmicas del producto final; afecta significativamente su comportamiento reológico, ajustándose al modelo de Ostwald-de Waale o ley de potencia, clasificando como seudoplástico y presentando tixotropía (Soukoulis et al., 2009). En el presente trabajo se analiza el efecto de la adición de salvado de trigo como fuente de fibra, en las propiedades reológicas del yogurt batido a base de leche de búfala.

\section{MATERIALES Y MÉTODOS}

Recolección y preparación de la materia prima. La leche de búfala se transportó hasta la planta piloto (taller de lácteos), en la sede Berástegui de la Universidad de Córdoba, en cantinas de aluminio, previamente lavadas y desinfectadas. Se utilizaron para cada tratamiento 5 litros de leche, se filtraron utilizando filtros desechables de papel, estandarizando a un porcentaje de grasa del $2,8 \%$ y posteriormente refrigerada hasta su uso. A la leche de Búfala estandarizada se le realizaron los análisis que se muestran en la Tabla 1.

Elaboración del yogurt. Para la elaboración del yogurt batido a partir de la leche de Búfala se utilizó la metodología descrita por Tamine y Robinson (1991), estandarizando la leche a un $2,8 \%$ de grasa y sin aumento del extracto seco (Fig. 1), debido que para mejorar la consistencia y textura del yogurt a partir de leche de búfala no es necesario la adición de leche en polvo (Verruma y Salgado, 1994). Se utilizó un cultivo liofilizado de adición directa que contiene Lactobacillus delbrueckii spp. bulgaricus y Streptococcus salivarius spp. Thermophilus; el salvado de trigo fue obtenido de los supermercados de la ciudad de Montería; reduciéndole el tamaño hasta un diámetro de $50 \mu \mathrm{m}$, utilizando un martillo de cizalla y adicionado durante el rompimiento del gel. 
Tabla 1: Análisis para la evaluación de la Leche de Búfala.

\begin{tabular}{|l|l|l|}
\hline \multicolumn{1}{|c|}{ Análisis } & \multicolumn{1}{c|}{ Método } & \multicolumn{1}{c|}{ Referencia } \\
\hline Toma de muestra & Muestra de 250-500 ml & A.O.A.C. 970.26 (1990) \\
\hline Materia Grasa & Método electrónico & FIL - IDF 141 B (1998) \\
\hline Antibióticos & Test colorimétrico (Devoltest) & Devoltest \\
\hline Sólidos Totales & Método electrónico & FIL - IDF 141 B (1998) \\
\hline Acidez & Método titrimétrico & A.O.A.C. 947.05 (1990) \\
\hline pH & Valoración potenciométrica & A.O.A.C. 973.41 (1990) \\
\hline
\end{tabular}

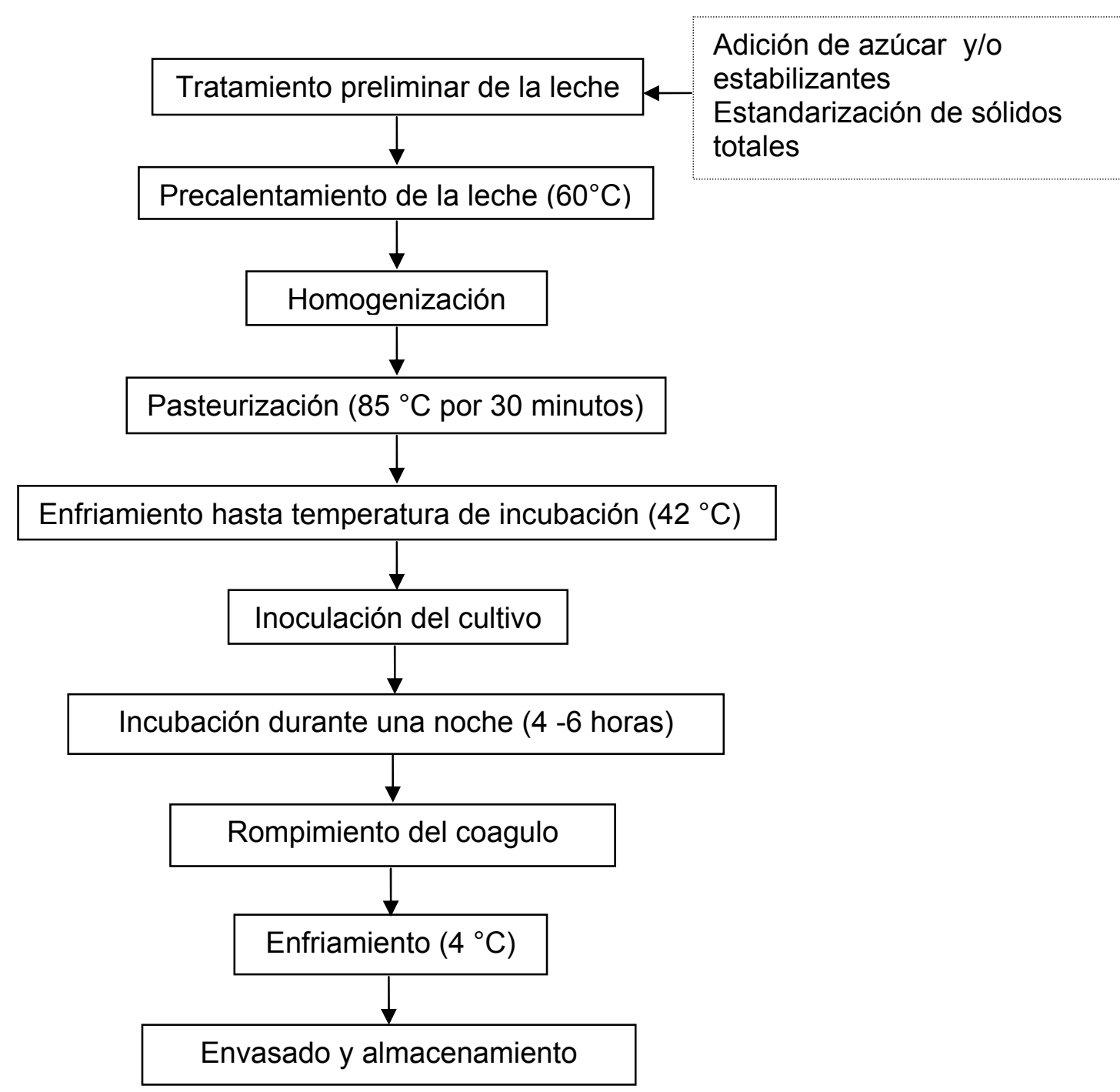

Fig. 1: Flujograma del proceso de elaboración del yogurt entero batido

Evaluación reológica del yogurt. Los parámetros reológicos del yogurt batido de leche de búfala se determinaron utilizando un viscosímetro Brookfied modelo DV-II+Pro, colocando la muestra en un adaptador con cilindros concéntricos, utilizando el spindle 21. Se varió el gradiente de cizalladura y determinando el esfuerzo cortante, las medidas se hicieron en forma ascendente hasta el gradiente más alto $\left(186 \mathrm{~s}^{-1}\right)$, donde se mantuvo por 2 minutos y después se disminuyó continuamente hasta el valor inicial. Los datos experimentales se ajustaron al modelo de Ostwald de Waele (ley de potencia). 
Diseño experimental. Se utilizó un diseño completamente al azar, bajo una estructura de tratamiento simple con cuatro tratamientos $(0,1,2$ y $3 \%$ de salvado de trigo), evaluándolo a los $0,7,14$ y 21 días de almacenamiento, realizando tres repeticiones por tratamiento. Los resultados se analizaron estadísticamente por análisis de varianza (ANOVA) y fue aplicado un test de comparación de Tukey $(p<0,05)$, utilizando el software STATISTICA versión 6.0 .

\section{RESULTADOS Y ANÁLISIS}

Caracterización fisicoquímica de la leche de Búfala. En la tabla 2, se presentan los valores promedio de algunos componentes fisicoquímicos de la leche de búfala estandarizada.

Tabla 2: Análisis fisicoquímico de la leche de búfala estandarizada

\begin{tabular}{|l|c|c|}
\hline \multicolumn{1}{|c|}{ Parámetro } & Promedio & Desviación estándar \\
\hline Acidez (\%Acido Lactico) & 0,192 & 0,0075 \\
\hline $\mathrm{pH}$ & 6,725 & 0,0217 \\
\hline Grasa $(\% \mathrm{~m} / \mathrm{v})$ & 2,767 & 0,1211 \\
\hline Sólidos totales $(\% \mathrm{~m} / \mathrm{m})$ & 13,398 & 0,0910 \\
\hline Densidad $(\mathrm{g} / \mathrm{ml})$ & 1,0405 & 0,00105 \\
\hline
\end{tabular}

Comportamiento reológico. Los datos reológicos se ajustaron adecuadamente $\left(R^{2} \geq 0,9783\right)$ a la ley de potencia o modelo de Oswald de Waele, el cual ha sido utilizado para predecir el comportamiento reológico en yogurt a partir de leche de vaca (Díaz et al., 2004), yogurt adicionado con lactosuero y transglutaminasa (Gauche et al., 2009), yogurt de leche de soya (Donkor et al., 2007), leches acidificadas (Janhøj et al., 2008) y leche concentrada de 25 a 30,5\% p/p (Vélez-Ruiz y BarbosaCánovas, 1998). Los modelos de Oswald de Waele, Herschel-Bulkley y Casson, son los tres más utilizados para caracterizar fluidos alimenticios no newtonianos (Mullineux y Simmons, 2008). El yogurt de leche de búfala con adición de salvado de trigo, en los diferentes tiempo de almacenamiento, se comporta como un fluido seudoplástico, índice de flujo (n) menor que 1 (Tabla $3)$.

Tabla 3: Parámetros reológicos del yogurt de leche de búfala

\begin{tabular}{|c|l|l|l|l|l|}
\hline Salvado de trigo, $\%$ & Parámetro & Día 0 & Día 7 & Día 14 & Día 21 \\
\hline \multirow{4}{*}{0} & $\mathrm{~K}_{\text {ascenso }}$ & 4,324 & 3,953 & 3,407 & 2,205 \\
\cline { 2 - 6 } & $\mathrm{K}_{\text {descenso }}$ & 2,266 & 2,138 & 2,028 & 1,879 \\
\cline { 2 - 6 } & $\mathrm{n}_{\text {ascenso }}$ & 0,534 & 0,610 & 0,658 & 0,797 \\
\cline { 2 - 6 } & $\mathrm{n}_{\text {descenso }}$ & 0,594 & 0,660 & 0,697 & 0,847 \\
\hline \multirow{4}{*}{1} & $\mathrm{~K}_{\text {ascenso }}$ & 4,385 & 3,645 & 2,978 & 2,880 \\
\cline { 2 - 6 } & $\mathrm{K}_{\text {descenso }}$ & 2,866 & 2,605 & 2,590 & 2,456 \\
\cline { 2 - 6 } & $\mathrm{n}_{\text {ascenso }}$ & 0,548 & 0,685 & 0,726 & 0,782 \\
\cline { 2 - 6 } & $\mathrm{n}_{\text {descenso }}$ & 0,626 & 0,746 & 0,772 & 0,808 \\
\hline \multirow{4}{*}{2} & $\mathrm{~K}_{\text {ascenso }}$ & 5,291 & 5,385 & 4,819 & 3,941 \\
\cline { 2 - 6 } & $\mathrm{K}_{\text {descenso }}$ & 3,223 & 3,234 & 2,907 & 2,978 \\
\cline { 2 - 6 } & $\mathrm{n}_{\text {ascenso }}$ & 0,577 & 0,637 & 0,788 & 0,790 \\
\cline { 2 - 6 } & $\mathrm{n}_{\text {descenso }}$ & 0,587 & 0,671 & 0,816 & 0,838 \\
\hline \multirow{3}{*}{3} & $\mathrm{~K}_{\text {ascenso }}$ & 5,106 & 4,159 & 4,408 & 3,725 \\
\cline { 2 - 6 } & $\mathrm{K}_{\text {descenso }}$ & 3,850 & 3,828 & 3,339 & 2,824 \\
\cline { 2 - 6 } & $\mathrm{n}_{\text {ascenso }}$ & 0,661 & 0,758 & 0,741 & 0,784 \\
\cline { 2 - 6 } & $\mathrm{n}_{\text {descenso }}$ & 0,694 & 0,793 & 0,742 & 0,875 \\
\hline
\end{tabular}


Este comportamiento ha sido reportado para yogurt adicionado con lactosuero y transglutaminasa (Gauche et al., 2009), yogurt a partir de leche de vaca (Díaz et al., 2004), yogurt de leche de maní (Isanga y Zhang, 2009), leche concentrada de 25 a 30,5\%p/p (Vélez-Ruiz y Barbosa-Cánovas, 1998) y helados con adición de fibra (Soukoulis et al., 2009); por lo que el yogurt de leche de búfala va disminuyendo su viscosidad aparente a medida que aumenta el gradiente de cizalladura, este comportamiento podría ser debido a la ruptura de la estructura del gel (Yu et al., 2007).

Los reogramas del yogurt de leche de búfala (Fig. 2) a diferentes concentraciones de salvado de trigo y tiempo de almacenamiento, presentan un área entre las curvas de ascenso y descenso (histéresis), indicando que el yogurt elaborado a partir de leche de búfala presenta propiedades reológicas dependiente del tiempo y se clasifica como un fluido tixotrópico, debido a que la viscosidad aparente disminuye con el tiempo de aplicación, al mismo gradiente de deformación. Esto coincide con lo reportado para arequipe (Rovedo et al., 1991), yogurt a partir de leche de vaca (Díaz et al., 2004) y helados con adición de fibra (Soukoulis et al., 2009). Alvarado (1996) sostiene que este carácter tixotrópico se debe a que existe un debilitamiento de la estructura y cambios de la consistencia conforme transcurre el tiempo. Este comportamiento tixotrópico es corroborado con el análisis de varianza realizado para el índice de consistencia $(K)$ y el índice de flujo $(n)$, que mostró que existían diferencias significativas entre los valores promedios de $\mathrm{K}$ y $\mathrm{n}$, en las lecturas de ascenso y descenso y en las diferentes concentraciones de fibra adicionada y tiempo de almacenamiento.
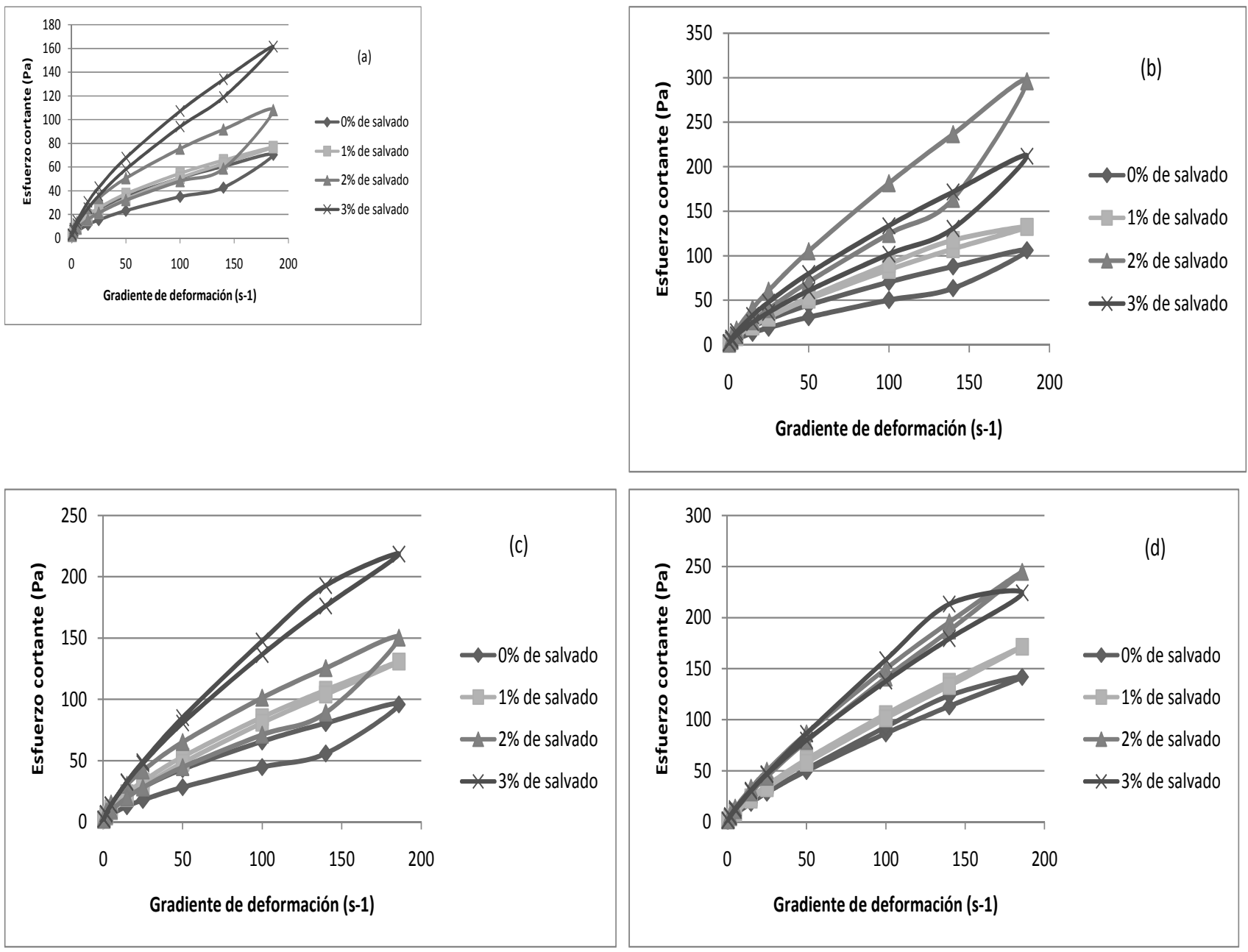

Fig. 2: Reogramas del yogurt de leche de búfala con (a) 0 día, (b) 7 días, (c) 14 días y (d) 21 días de almacenamiento.

Efecto de la adición de salvado de trigo sobre los parámetros reológicos. Considerando las diferencias significativas entre los tratamientos, se aplicó una prueba de Tukey para el índice de consistencia y el índice de flujo (Tabla 4). La adición de fibra en la elaboración de yogurt a partir de 
leche de búfala, influyó de manera directamente proporcional sobre el índice de consistencia y el índice de flujo. Igual comportamiento tiene la adición de fibra en el yogurt a partir de leche de vaca (Díaz et al., 2004). Por lo cual, la adición de salvado de trigo en la elaboración de yogurt a partir de leche de búfala, hace que este se torne más consistente y menos seudoplástico.

Efecto del tiempo de almacenamiento sobre los parámetros reológicos. Considerando las diferencias significativas entre los tratamientos, se aplicó una prueba de Tukey para el índice de consistencia y el índice de flujo (Tabla 5). El tiempo de almacenamiento en la elaboración de yogurt a partir de leche de búfala, influyó de manera inversamente proporcional sobre el índice de consistencia y directamente proporcional sobre el índice de flujo. Igual comportamiento tiene la adición de fibra en el yogurt a partir de leche de vaca (Díaz et al., 2004), yogurt batido adicionado con pectina (Ramaswamy y Basak, 1992) y con frutas concentradas (Basak y Ramaswamy, 1994). Por lo cual, el tiempo de almacenamiento en la elaboración de yogurt a partir de leche de búfala, hace que este se torne menos consistente y seudoplástico, con una tendencia a ser un fluido Newtoniano. Esto se puede deber a la perdida de firmeza de la matriz proteica, mayor sinéresis y perdida de la consistencia.

Tabla 4: Tukey para la adición de salvado de trigo

\begin{tabular}{|c|c|c|}
\hline Tratamiento & K mean $^{\mathrm{n}}$ & $\mathrm{n}$ mean \\
\hline 1 & $2,775148^{\mathrm{a}}$ & $0,674602^{\mathrm{a}}$ \\
\hline 2 & $3,050606^{\mathrm{a}}$ & $0,711443^{\mathrm{a}}$ \\
\hline 3 & $3,872413^{\mathrm{b}}$ & $0,712934^{\mathrm{a}}$ \\
\hline 4 & $3,904807^{\mathrm{b}}$ & $0,756054^{\mathrm{b}}$ \\
\hline
\end{tabular}

Tabla 5: Tukey para el tiempo de almacenamiento

\begin{tabular}{|c|l|l|}
\hline Tiempo, día & K mean & $\mathrm{n}$ mean \\
\hline 0 & $3,913903^{\mathrm{a}}$ & $0,602507^{\mathrm{a}}$ \\
\hline 7 & $3,618479^{\mathrm{ab}}$ & $0,695080^{\mathrm{b}}$ \\
\hline 14 & $3,309431^{\mathrm{b}}$ & $0,742419^{\mathrm{c}}$ \\
\hline 21 & $2,861161^{\mathrm{c}}$ & $0,815026^{\mathrm{d}}$ \\
\hline
\end{tabular}

\section{CONCLUSIONES}

El yogurt elaborado a partir de leche de búfala hasta con el $3 \%$ de salvado de trigo y hasta un tiempo de almacenamiento de 21 días, se comporta como un fluido seudoplástico y tixotrópico. La adición de salvado de trigo hace que el yogurt sea más consistente y menos seudoplástico y el tiempo de almacenamiento lo torna menos consistente y seudoplástico, con una tendencia a ser un fluido newtoniano.

\section{REFERENCIAS}

Alvarado, J., Principios de ingeniería aplicados a alimentos (en línea). Secretaria general de la O.E.A. Programa regional de desarrollo científico y tecnológico. Quito (1996). http://www.science.oas.org/simbiol/prin_ali/principios.pdf. Acceso: 24 marzo (2008).

A.O.A.C. Oficial method of analysis. Association of Official Analytical Chemistry. 16 th edition, ed. By Hoorwitz, N., Chialo, P. and Reynolds, H. Benjamín Franklin., Station, Washington (1990). 
Basak, S. y H. S. Ramaswamy, Simultaneous evaluation of shear rate and time dependency of stirred yogurt rheology as influenced by added pectin and strawberry concentrate. Journal of food Engineering: 21(3), 385-393 (1994).

Díaz, B., M. Sosa, y J. Vélez, Efecto de la adición de fibra y la disminución de grasa en las propiedades fisicoquímicas del yogur. Revista Mexicana de Ingeniería Química: 3, 287-305 (2004).

Donkor, O., A. Henriksson, T. Vasiljevic, y N. P. Shah, Rheological properties and sensory characteristics of set-type soy yogurt. Journal of Agricultural and Food Chemistry: 55(24), 9868-9876 (2007).

FIL-IDF Standard 141 B (1998): Whole milk.Determination of milkfat, protein and lactosecontent. Guide for the operation of mid-infra-redinstruments. Brussells, Belgium.

Gauche, C., y otros cuatro autores, Physical properties of yoghurt manufactured with milk whey and transglutaminase. LWT - Food Science and Technology: 42(1), 239-243 (2009).

Guarín, J.; Grupo de Estudio sobre Búfalos. www.bufaloscolombia.com, Colombia (2002). Acceso: 10 diciembre (2008).

Isanga, J. y G. Zhang, Production and evaluation of some physicochemical parameters of peanut milk Yoghurt. LWT - Food Science and Technology 42(6), 1132-1138 (2009).

Janhøj, T., M. Frøst, y R. Ipsen, Sensory and rheological characterization of acidified milk drinks. Food Hydrocolloids: 22(5), 798-806 (2008).

Mullineux, G. y M. Simmons, Influence of rheological model on the processing of yogurt. Journal of Food Engineering: 84(2), 250-257 (2008).

Patiño, E., La leche de Búfala. Visión Revista electrónica de veterinaria. ISSN 1695-7504 (en línea), 4(3), 2004. http://www.visionveterinaria.com. Acceso: 10 de diciembre (2008)

Ramaswamy, H.S. y S. Basak, Pectin and raspberry concentrate effects on the rheology of stirred commercial yogurt. Journal of Food Science: 57(2), 357-360 (1992).

Razavi, S. y H. Karazhiyan, Flow properties and thixotropy of selected hydrocolloids: Experimental and modeling studies. Food Hydrocolloids: 23(3), 908-912 (2009).

Rovedo, C., P. Viollaz, y C. Suarez, The effect of $\mathrm{pH}$ and temperature on the rheological behavior of dulce de leche, a typical dairy Argentine. Product Journal Dairy Science: 74(5), 1497-1502 (1991).

Saxelin, M., R. Korpela, y A. Mäyrä-Makiren, Introduction: classifying functional dairy products. In Functional Dairy Products, pp. 1-16, CRC Press. Cambridge, Inglaterra (2003)

Silva, M., y otros 6 autores; Programa de incentivo a criação de búfalos por pequenos produtores PRONAF. (2003). http://www.cpatu.Silva et al, 2003.br/bufalo. Acceso 10 de diciembre (2008).

Soukoulis C., D. Lebesi, y C. Tzia, Enrichment of ice cream with dietary fibre: Effects on rheological properties, ice crystallisation and glass transition phenomena. Food Chemistry: 115(2), 665-671 (2009).

Tamime, A. y R. Robinson, Yogurt Ciencia y Tecnología, 368. Acribia, Zaragoza, España. (1991).

Vélez, J. y A. Rivas, Propiedades y características del yogur. Revista Internacional Información Tecnológica: 12 (6), 35-42 (2001).

Vélez-Ruiz, J.F. y G.V. Barbosa-Cánovas, Rheological properties of concentrated milk as a function of concentration, temperature and storage time. Journal of Food Engineering: 35(2), 177-190 (1998).

Verruma, M. y J. Salgado, Avaliação nutricional do leite de búfala em comparação ao leite de vaca, Scientia Agrícola: 51(1), 131-137 (1994).

Yu, J., M. Ahmedna, y I. Goktepe, Peanut protein concentrates: production and functional properties as affected by processing. Food Chemistry: 103(1), 121-129 (2007). 
